\title{
NOVAS TENDÊNCIAS DE COMBATE AO BIOFILME DE CANDIDA EM PRÓTESES DENTÁRIAS
}

\author{
NEW TRENDS TO COMBAT CANDIDA BIOFILM IN DENTAL \\ PROSTHETICS
}

\author{
Vanessa Zafaneli Bergamoํ, Daiane Flores Dalla Lana², Bruna Pippi ${ }^{1}$, \\ Irene Clemes Kulkamp Guerreiro², Alexandre Meneghello Fuentefria ${ }^{1,2}$
}

\section{RESUMO}

Apesar de a espécie Candida albicans ser efetivamente o microrganismo mais frequentemente associado a estomatite protética, as espécies de Candida não albicans já foram isoladas nas superfícies de dentaduras e da mucosa oral de indivíduos com essa lesão eritematosa. A virulência das espécies de Candida e a capacidade de adesão a polímeros acrílicos são condições prévias para a colonização e o desenvolvimento de biofilmes em superfícies de dentaduras. Estudos recentes focam na tentativa de modificação das resinas acrílicas para diminuir a adesão de cepas patogênicas e formadoras de biofilme do gênero Candida spp. Dentro desse aspecto, esta revisão sistematiza o atual panorama epidemiológico da estomatite protética associada ao uso de próteses dentárias, bem como as atuais e novas opções de combate ao biofilme fúngico especializado na adesão desse tipo de biomaterial.

Palavras-chave: Candida spp.; biofilme; estomatite protética; resina acrílica; tratamento

\begin{abstract}
Although the Candida albicans species is effectively the microorganism most frequently associated with prosthetic stomatitis, Candida non-albicans species have already been isolated from the denture and oral mucosal surfaces of individuals with this erythematous lesion. The virulence of Candida species and the ability to adhesion to acrylic polymers are preconditions for the colonization and development of biofilms on denture surfaces. Recent studies focus on the attempt to modify the acrylic resins to reduce the adhesion of pathogenic and biofilm forming strains of the genus Candida spp. In this aspect, this review systematizes the current epidemiological panorama of prosthetic stomatitis associated with the use of dental prostheses, as well as new options for combating the fungal biofilm specialized in the adhesion of this type of biomaterial.
\end{abstract}

Keywords: Candida spp.; biofilm; prosthetic stomatitis; acrylic resin; treatment

Candida albicans é o agente etiológico mais prevalente de candidoses eritematosas crônicas, conhecidas como estomatite protética (EP). Essa lesão eritematosa é uma reação inflamatória da mucosa palatina e alveolar subjacente à dentadura removível. A EP é diagnosticada em 25 a $42 \%$ dos idosos portadores de próteses dentárias ${ }^{1}$. Contudo, a incidência dessa condição aumenta para mais de $76 \%$ quando o paciente usa o mesmo biomaterial por mais de 10 anos $^{1}$. Essa enfermidade possui etiologia multifatorial, sendo que a má higiene e o uso contínuo de próteses dentárias têm sido as causas mais frequentemente associadas a essa doença².

Apesar de C. albicans ser o microrganismo mais recorrentemente associado a EP, as espécies de $C$. não albicans (CNA) já foram isoladas das superfícies de dentaduras de pacientes com essa enfermidade ${ }^{3,4}$. As próteses dentárias proporcionam superfícies que facilitam a acumulação de biofilmes de placas ao longo do tempo. O biofilme de Candida spp. na resina acrílica
Clin Biomed Res. 2018;38(2):155-166

1 Programa de Pós-graduação em Microbiologia Agrícola e do Ambiente, Departamento de Análises, Faculdade de Farmácia, Universidade Federal do Rio Grande do Sul (UFRGS). Porto Alegre, RS, Brasil.

2 Programa de Pós-graduação em Ciências Farmacêuticas, Departamento de Análises, Faculdade de Farmácia, Universidade Federal do Rio Grande do Sul (UFRGS). Porto Alegre, RS, Brasil.

Autor correspondente: Vanessa Zafaneli Bergamo bergamovanessa@hotmail.com Programa de Pós-graduação em Microbiologia Agrícola e do Ambiente, Departamento de Análises, Faculdade de Farmácia, Universidade Federal do Rio Grande do Sul (UFRGS)

Av. Ipiranga, 2752.

90610-000, Porto Alegre, RS, Brasil. 
se desenvolve através da adesão, que ocorre de forma direta na superfície condicionada ou através de uma camada de placa de dentadura preexistente ${ }^{5}$. Muitos fatores que afetam a adesão de Candida spp. foram descritos, incluindo interações hidrofóbicas e rugosidade da superfície de acrílico dos materiais protéticos ${ }^{6}$.

No que tange à virulência de Candida, a capacidade de adesão ao acrílico é uma condição prévia para a colonização e o desenvolvimento de biofilmes em superfícies de dentaduras. Portanto, a inibição da adesão poderia ser eficaz para tratar ou prevenir a EP ${ }^{6}$. Alguns estudos focam na tentativa de modificação das resinas acrílicas para diminuir o fator de adesão de Candida spp., ${ }^{6,7}$. Dessa forma, vêm sendo utilizadas modificações químicas da carga superficial das resinas como recurso para retardar ou prevenir o efeito da adesão leveduriforme ${ }^{6,8,9}$.

Esta revisão sistematiza o atual panorama epidemiológico da EP associada ao uso de próteses dentárias, bem como as atuais e novas opções de combate ao biofilme fúngico especializado na adesão desse tipo de biomaterial.

\section{MÉTODOS}

Foi realizado um estudo de revisão bibliográfica sistemática a partir de bancos de dados e coleções da biblioteca virtual de saúde do National Institutes of Health (PubMed) e do Portal de Periódicos da Coordenação de Aperfeiçoamento de Pessoal de Nível Superior do Ministério da Educação (CAPES/MEC), no período de janeiro a outubro de 2017. A busca eletrônica foi realizada através dos termos "biofilme/biofilm", "Candida não-albicans/Candida non-albicans", "resina acrílica/acrylic resin", tratamento/treatment", "estomatite protética/stomatitis prosthetic", epidemiologia/epidemiology". Nesse contexto, a revisão teve como abordagem os aspectos mais relevantes que contemplam a formação de biofilme fúngico quando relacionado à EP em pacientes que utilizam próteses dentárias, a terapêutica convencional e novos estudos que vêm sendo realizados para o tratamento dessa doença. Os critérios de inserção foram artigos originais e de revisão que se enquadrassem na temática estabelecida publicados entre 2007 e-2017. Foram eliminados os estudos que possuíam referências bibliográficas inadequadas, incompletas ou com fontes terciárias. Dissertações, teses, monografias e livros não foram incluídos. Assim, 72 artigos atenderam a todos os critérios acima mencionados e foram detalhadamente analisados para fazerem parte deste estudo.

\section{Candida spp. em Próteses Dentárias e Patologias Associadas}

Devido ao aumento da longevidade, é cada vez maior a população senil em todo o mundo. No Brasil, o século XXI iniciou com o crescimento da população idosa quase oito vezes maior quando comparado ao dos jovens ${ }^{10}$. Os pacientes edêntulos necessitam de reabilitação protética, com próteses orientadas biologicamente que correspondam às necessidades reais do indivíduo, promovendo o conforto e a mastigação adequada ${ }^{11}$.

Kumaresan et al. ${ }^{12}$ realizaram um estudo de revisão abordando a síndrome da boca ardente, caracterizada pela sensação de queimação, picada e/ou prurido da cavidade oral na ausência de lesão oral específica. Essa circunstância afeta principalmente mulheres de meia-idade e idosas com alterações hormonais ou distúrbios psicológicos. O paciente que possui essa síndrome pode apresentar distúrbios gustativos e xerostomia subjetiva. Essa condição é provavelmente de origem multifatorial, envolvendo várias causas locais, sistêmicas e/ou psicogênicas. Gênero feminino, pré-menopausa, climatério, depressão, ansiedade, doença de Parkinson e condições médicas crônicas, incluindo doenças gastrointestinais e urogenitárias, são fatores de risco para desenvolver essa enfermidade. Ainda na revisão de Kumaresan et al. ${ }^{12}$, as infecções orais foram investigadas e o gênero Candida foi identificado como sendo mais prevalente em pacientes com a síndrome da boca ardente.

O paciente que utiliza prótese e que sofre de xerostomia tem risco maior de patologia oral e modificações da função oral, devido à retenção não adequada da prótese ${ }^{11}$. Somam-se a isso queixas de halitose, ardência bucal e intolerância a sabores ácidos. A reabsorção óssea pode originar desadaptação na prótese e o desgaste dos dentes nela incluídos pode diminuir a altura da face ${ }^{11}$. As próteses mandibulares, quando empregadas, tendem a estar mais desadaptadas que as próteses maxilares ${ }^{13}$.

O predomínio das lesões orais no adulto já foi evidenciado a nível mundial em países como Colômbia, México, Brasil, Chile, Argentina, EUA, Israel, e em outros países asiáticos ${ }^{14}$. Em um estudo realizado na Venezuela, 340 pacientes foram avaliados clinicamente e, destes, $50 \%$ apresentaram uma ou mais lesões orais, principalmente associadas ao uso de prótese dentária. As lesões foram mais frequentemente observadas nos grupos de pessoas entre 60 a 74 anos e a alteração mais observada foi EP ${ }^{14}$.

Bianchi et al. ${ }^{15}$ realizaram um estudo transversal em que foram coletadas amostras de saliva de 48 pacientes 
portadores de próteses dentárias e de 43 pacientes (grupo controle) que não utilizavam prótese. Como resultado, entre os 91 pacientes, Candida spp. foi isolada em $40(83,3 \%)$ que utilizavam próteses e em $23(53,5 \%)$ do grupo controle. O agente etiológico mais frequentemente encontrado foi $C$. albicans (37 isolados), sendo mais prevalente no grupo representado pelos portadores de próteses dentárias $(n=23 ; 62,2 \%)$ do que no grupo controle $(n=14 ; 37,8 \%)$. Além disso, entre os paciente que apresentavam manifestações clínicas de candidíase oral $(n=24), 83,3 \%(n=20)$ pertenciam ao grupo que usava prótese dentária, enquanto que apenas $16,7 \%(n=4)$ pertenciam ao grupo controle. Curiosamente, os pacientes idosos com diabetes apresentaram risco estimado 4,4 vezes maior de desenvolver candidíase oral quando comparados com indivíduos sem diabetes. Nesse estudo não houve associação estatisticamente significante entre ser usuário de próteses e ter diabetes com o início de candidíase. $O$ autor sugere que o uso de próteses e a falta de higiene bucal em pacientes idosos predispõem ao desenvolvimento da candidíase oral ${ }^{15}$.

A candidíase oral, também conhecida como estomatite cremosa ou popularmente como "sapinho", é um processo infeccioso ocasionado pelos fungos do gênero Candida. Parte das manifestações da enfermidade varia de acordo com a região acometida pela infecção, que pode ser: mucocutânea (entra em contato com a mucosa oral e vaginal), cutânea e sistêmica. Pode ser causada por diferentes espécies do gênero, tais como C. tropicalis, C. glabrata, C. krusei, C. parapsilosis e C. albicans ${ }^{16}$. Nos pacientes que utilizam próteses, é denominada EP, candidíase atrófica ou estomatite por prótese ${ }^{2}$.

AEP é uma doença crônica associada com o uso de próteses dentárias que pode ser evidenciada por uma inflamação localizada ou generalizada da mucosa bucal e que apresenta tratamento complexo, devido à etiologia multifatorial. Utilização contínua das próteses, porosidade da resina acrílica, alergia ao monômero residual, formação de biofilme, hipossalivação e falta de higienização são as etiologias mais comuns relacionadas a EP, $2,5,17-19$.

Como já mencionado, a candidíase atrófica crônica é comumente designada estomatite protética, sendo observada em pacientes que utilizam próteses removíveis mal ajustadas por períodos prolongados, principalmente pelo fato de não removê-las à noite ${ }^{20}$.

AEP pode ser classificada em três tipos, conforme proposto por Newton ${ }^{21}$ :

- Tipo I: Hiperemia puntiforme (classe I) É determinada pela hiperemia dos ductos de glândulas salivares palatinas menores, o que confere aparência eritematosa puntiforme, podendo ocupar áreas dispersas ou pequenas áreas localizadas no palato;

- Tipo II: Hiperemia difusa (classe II) - É o tipo mais frequentemente encontrado, caracterizando-se pela presença de mucosa lisa e atrófica, com aspecto eritematoso em toda a localidade sob a prótese;

- Tipo III: Hiperemia granular (classe III) - É comumente associada à câmara de sucção, acometendo a região central do palato com aspecto clínico nodular e rugoso da mucosa.

Além disso, os pacientes com estomatite apresentam usualmente quelite angular associada ${ }^{20}$. Essa variante da candidíase representa uma combinação de fungos e infecções bacterianas. A maioria dos casos de quelite angular são ocasionados por $C$. albicans e Staphylococcus aureus. A forma clínica apresentada pelo paciente inclui dor, eritema e fissura nas comissuras, na maioria das vezes de forma bilateral. Essa enfremidade acomete pacientes portadores de próteses dentárias, especialmente aqueles com uma perda da dimensão vertical de oclusão. A quelite inclui deficiências nutricionais como ferro, vitamina B12, ácido fólico, tiamina, e riboflavina. Essa lesão também é notada em pacientes dentados mais jovens que são HIV positivo, devido à diminuição da imunidade ${ }^{20}$.

Arnaud et al. referiram que, embora o tecido apresente-se hiperêmico e inflamado, a EP é frequentemente assintomática e, por isso, passa muitas vezes despercebida pelos usuários, sendo inesperadamente descoberta durante exame bucal de rotina. Contudo, a mucosa do paciente pode apresentar hemorragia, prurido, queimação, desconforto, sabor desagradável, halitose e xerostomia ${ }^{22}$.

A EP comumente não é uma condição que traz agravos sérios ao usuário; contudo, a mucosa inflamada se torna um sustentáculo deficiente para a prótese, dificultando seu uso. A EP é considerada uma lesão bucal repetida de fundamental importância a ser diagnosticada e tratada para melhoria da qualidade de vida dos pacientes acometidos ${ }^{23}$.

\section{BIOFILME}

\section{Espécies de Candida Formadoras de Biofilme em Resinas Acrílicas}

No ser humano, a cavidade oral é um reservatório de aproximadamente 700 espécies de microrganismos, sendo 20 dessas Candida spp. ${ }^{11,24}$. Esse microrganismo leveduriforme não é prejudicial em seres humanos saudáveis, mas pode ocasionar infecções oportunistas 
em pacientes imunocomprometidos, resultando em candidíase ${ }^{25}$ principalmente na idade senil, quando se faz necessário o uso de próteses artificiais que causam mudanças no ambiente e na flora oral ${ }^{26}$.

A patogenicidade da Candida foi associada a diversos fatores de virulência, englobando adesão a células hospedeiras ou dispositivos médicos e secreção de enzimas hidrolíticas, como proteases, fosfolipases e hemolisinas ${ }^{27,28}$. Além disso, os principais fatores determinantes do curso infeccioso são saliva, formação de biofilmes e natureza do substrato, que pode variar individualmente e em relação ao tipo de microrganismo ${ }^{2}$. Entre as principais características das superfícies da prótese que favorecem a adesão desses microrganismos, ressalta-se a hidrofobicidade e a rugosidade superficial.

As próteses totais podem possibilitar uma superfície dura sem manchas em um ambiente estagnado para os microrganismos atacarem e colonizarem. Dessa forma, a dentadura pode proporcionar um reservatório de colonização e consequente infecção. O local da prótese na mucosa oral torna a ligação da superfície menos disponível ao fluxo salivar ou inacessível à ação mecânica da língua, modificando as condições do meio ambiente. Assim, ocorre a propagação e o crescimento de Candida spp. na área entre a prótese dentária e a mucosa portadora da prótese 29-32. $^{2}$.

A rugosidade da prótese é um fator importante na adesão de microrganismos, como já mencionado. Esse parâmetro pode ser avaliado através do Ra (média aritmética dos valores absolutos das ordenadas de afastamento dos pontos de perfil de rugosidade em relação à linha média do percurso de medição expressa em $\mu \mathrm{m}$ ). Em seus estudos, Nevzatoğlu et al ${ }^{33}$ avaliaram a rugosidade e a aderência de $C$. albicans na superfície de resinas acrílicas para base de dentadura e reembasadores com diferentes acabamentos da superfície (vidro, gesso, broca de tungstênio). Observou-se que a rugosidade foi menor nas resinas acrílicas quando comparada à das bases resilientes e que a adesão de $C$. albicans foi menor em superfícies de vidro. Já as brocas de tungstênio apresentaram maiores valores, enquanto que não houve diferença estatística das superfícies dos espécimes processados em gesso. Como a superfície interna das próteses não possui facilidade de ser polida mecanicamente, é considerado um campo crítico para o desenvolvimento de processos patológicos, devido ao contato com os tecidos de suporte e sua topografia favorecer um acréscimo da área de nichos de retenção que favorecem a aderência de microrganismos e a formação de biofilme ${ }^{34}$.
Em 2009, Subramani et al. ${ }^{35}$ realizaram uma revisão da literatura (de 1966 e junho de 2007) referente à formação de biofilmes em implantes dentários e à influência das características de superfície (química, energia livre de superfície e rugosidade) dos materiais de implante e pilares protéticos, bem como seu desenho e formato, sobre a formação de biofilme e suas consequências. Como resultado do estudo, foi possível observar que o avanço da rugosidade e da energia livre de superfície promove a formação de biofilmes em superfícies de implantes dentários e em elementos protéticos ${ }^{35}$.

O biofilme pode colonizar qualquer tipo de prótese, e essa afinidade das próteses com o biofilme sugere que $65 \%$ das infecções microbianas envolvem a presença de células sésseis ${ }^{2}$. Na Região Centro-Oeste do Brasil (Goiás) foi realizada uma pesquisa ${ }^{36}$ para avaliar a colonização de leveduras do gênero Candida em 312 amostras de saliva de profissionais do Hospital das Clínicas da Universidade Federal de Goiás durante o período de julho a dezembro de 2007. Os profissionais apresentaram colonização por leveduras do gênero Candida em $61,9 \%$ das amostras analisadas, sendo isolados $C$. albicans $(58,5 \%)$, C. parapsilosis $(24,4 \%)$, C. krusei $(8,3 \%)$ e C. famata $(3,6 \%)$. Inicialmente a microbiota das próteses removíveis foi considerada semelhante à da placa dentária. Entretanto, estudos recentes demonstraram que o biofilme maduro apresentou menor contagem de microrganismos nos dentes quando comparados às próteses ${ }^{37}$.

A prótese dentária é composta por uma combinação de microrganismos (fungos e bactérias) e por células epiteliais que estão conectadas dentro de uma matriz mucopolissacarídica, ideal para a ligação da placa à superfície da prótese ${ }^{37}$.

Para a formação do biofilme, a prótese dentária com base de resina acrílica exibe carga positiva e alta energia de superfície. Isso faz com que as glicoproteínas salivares com carga negativa sejam ligeiramente atraídas, formando uma estrutura semelhante à película adquirida do dente, com intensa adesão química à prótese. A etapa seguinte é a colonização de microrganismos que também possuem carga negativa e se aproximam da película adquirida por meio de forças de Van der Waalls e se unem a ela por meio de adesinas e pontes de hidrogênio. Ocorre uma adesão mecânica beneficiada pelas irregularidades e porosidades da resina. As adesinas são enzimas encontradas nos microrganismos que apresentam receptores específicos na película adquirida ou nas células epiteliais da boca ${ }^{38,39}$. Diversos são os sintomas clínicos relatados pelos pacientes: dor, halitose, prurido e percepção de queimação podem acontecer pelo fato de a $C$. albicans produzir exoenzimas, 
constituídas principalmente por proteinases que facilitam a adesão do biofilme, e também devido a fatores do hospedeiro, tais como redução do $\mathrm{pH}$, do fluxo salivar e da microbiota oral ${ }^{40}$ (Figura 1).

Após a adesão, ocorre a proliferação das formas hifais em torno da superfície da prótese dentária e o desenvolvimento do complexo microbiano. A formação de biofilmes de $C$. albicans ocorre principalmente após 24-48 h de crescimento e ocorre em três fases: (1) adesão de blastósporos na superfície; (2) as leveduras se multiplicam para cobrir uma extensa área superficial e ocorre a produção dos polímeros extracelulares; e, por fim, (3) ocorre a fase de maturação ${ }^{41,42}$. Para as cepas de $C$. tropicalis e C. parapsilosis, é notória a presença de blastoconídios e pseudo-hifas. Ambas as espécies apresentam uma estrutura do biofilme em monocamada compacta não contínua; entretanto, em termos de carboidratos e proteínas, C. parapsilosis apresenta maior quantidade. $\mathrm{Na}$ espécie de C. parapsilosis há a presença de blastoconídios, e para a C. glabrata observam-se somente blastoconídios com expressiva quantidade de carboidratos e proteínas em uma estrutura do biofilme em monocamada compacta ou multicamada ${ }^{43}$.

Um fator importante de comunicação que ocorre entre os microrganismos dentro do biofilme é conhecido como moléculas quorum-sensing (MQS). MQS são moléculas autoindutoras que à medida que crescem proporcionam um aumento da população. Após alcançar um limiar crítico, é desenvolvida uma resposta regulatória de expressão/inibição que é monitorada por genes ${ }^{44}$.

Em 2009, Ten Cate et al..$^{45}$ observaram que as infecções fúngicas na cavidade bucal são ocasionadas principalmente por $C$. albicans. Entretanto, diversas outras espécies de Candida vem sendo identificadas, aumentando a sua prevalência em pacientes que utilizam próteses totais, o que ocasiona infecções invasivas com alta taxa de mortalidade.

Outros autores também sugerem que $C$. albicans ainda é considerada a espécie mais predominante e virulenta nos casos de EP, embora um aumento expressivo da prevalência de espécies de CNA vem sendo determinante de infecções em pacientes que utilizam próteses. Entre elas destacam-se C. glabrata, C. dubliniensis, C. parapsilosis, C. kruseie C. tropicalis ${ }^{19}$. Além disso, Prakash et al. ${ }^{25}$ constataram que a prevalência de diferentes espécies de Candida foi significativamente maior em usuários de próteses, sendo que as espécies mais predominantes foram C. albicans, C. tropicalis, C. dubliensis e C. glabrata. Evidencia-se que nesse caso a idade foi um fator relacionado à prevalência de Candida spp.

Pathak et al. ${ }^{46}$ realizaram um estudo utilizando a espécie de Candida (C. albicans, C. glabrata, C. krusei e C. tropicalis) e multiespécies da microbiota oral de pacientes neutropênicos. A produção do biofilme na resina acrílica foi avaliada pelo método cristal violeta. Foi relatado que houve diferenças entre a formação de biofilme de espécies individuais (C. glabrata> C. krusei> C. tropicalis> C. albicans) e em suas combinações de múltiplas espécies (a maior para C. albicans com C. glabrata e o menor para a combinação das quatro espécies).

Em 2013, Valentini et al. ${ }^{47}$ estudaram como o desenvolvimento e a constituição do biofilme são afetados pelo tempo e pelo tipo de material dentário em portadores de prótese (com e sem EP). Nesse ensaio foi utilizada a resina acrílica como controle e prótese dentária (à base de silicone ou de resina acrílica) na superfície intacta de 30 usuários de próteses. Como resultado, as contagens de espécies de CNA foram superiores em pacientes com próteses à base de silicone.

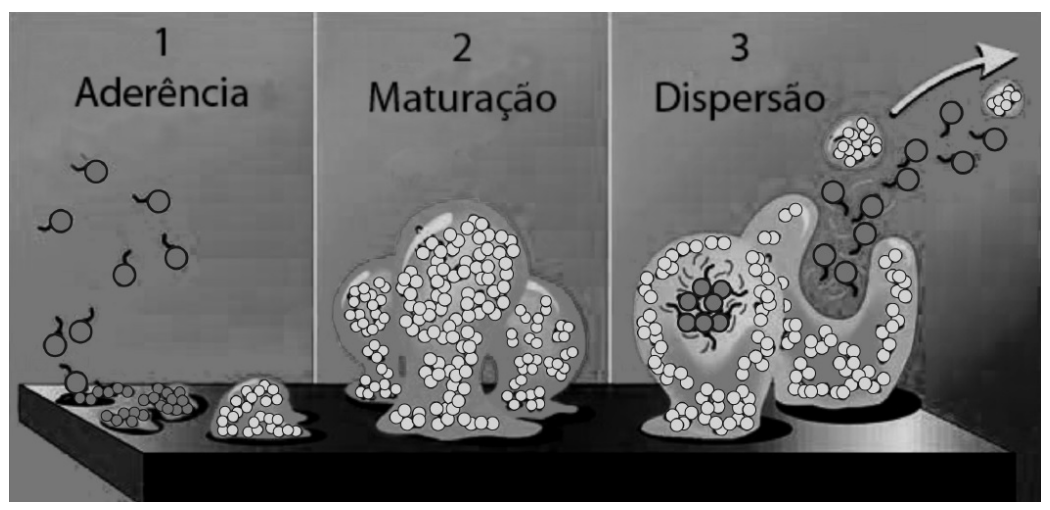

Figura 1: Esquema da formação do biofilme fúngico típico de Candida albicans. (1) Aderência do microrganismo ao substrato, quando as células se replicam e começam a secretar matriz extracelular e formar as pseudo-hifas; (2) O biofilme maduro é formado na presença de microcolônias e hifas e da matriz polimérica extracelular que envolvem as células; (3) As leveduras começam a dispersar do biofilme, disseminando a infecção, e reinicia-se o ciclo novamente. 
Sánchez-Vargas et al. ${ }^{48}$ avaliaram a produção de biofilme de espécies de Candida e observaram que $C$. albicans e, em menor expansão, CNA são comumente encontrados nas cavidades bucais de adultos e crianças e raramente causam doença. Sendo assim, isolados orais de C. glabrata foram produtores fortes de biofilme, enquanto $C$. albicans e C. tropicalis foram consideradas espécies produtoras moderadas.

Estudo recente publicado por Bergamo et al..$^{40}$ avaliou a formação do biofilme de espécies de CNA (C. tropicalis, C. glabrata, C. parapsilosis) e C. albicans em resina acrílica através da contagem de unidades formadoras de colônia/mL. Com base nos resultados, observou-se que a menor formação do biofilme foi da C. albicans, com maior predomínio das demais espécies.

\section{TRATAMENTO}

\section{Tratamento Convencional}

A busca por tratamentos pertinentes à utilização de próteses no Brasil tem se tornado mais evidente, devido aos elevados índices de edentulismo e às condições precárias de saúde bucal, indicando a necessidade da apropriação das próteses dentárias pela população. É importante mencionar que o tratamento básico de EP inclui a boa higiene bucal, procedimentos de limpeza da dentadura, antifúngicos tópicos ou sistêmicos, e, em casos extremos, substituição de prótese ${ }^{49}$.

O tratamento inserido para a Candida spp. é composto pelos principais grupos de fármacos, a saber: poliênicos (nistatina e anfotericina B), triazólicos (fluconazol, itraconazol, voriconazol, posaconazol), equinocandinas (caspofungina, micafungina, anidulafungina).

Em 2013, Skupien et al. ${ }^{50}$ realizaram uma revisão sistemática (1950-2012) que avaliou a viabilidade de prevenção para a adesão de Candida spp. em revestimentos dentários. Em sua revisão foi visto que, para previnir ou inibir a colonização por Candida spp. nas lesões de EP, a nistatina (500.000 unidades), hipoclorito de sódio $(0,5 \%)$ e a irradiação de microondas são os métodos alternativos mais relatados na literatura.

Em 2014, Peixoto et al. ${ }^{51}$ realizaram um estudo que avaliou o tratamento da candidíase, enfatizando que a nistatina é o farmaco de primeira escolha para o tratamento da EP devido à sua eficácia e ao custo reduzido em comparação aos demais fármacos. A nistatina deve ser administrada em doses de 200.000 a 600.000 unidades (três a quatro vezes por dia, com instrução para bochechar e deglutir). Além disso, outro fármaco frequentemente utilizado é o clotrimazol em pastilhas orais ou comprimidos (10 mg), que deve ser administrado por 14 dias (cinco vezes ao dia).

Em 2016, Iqbal e Zafar ${ }^{52}$ realizaram uma revisão sistemática e relataram a eficácia da adição de antifúngicos convencionais (nistatina, derivados do grupo azol e clorhexidina, antimicrobianos, zeólito de prata, nanopartículas de prata, fotocatalisadores, óxidos metálicos, óleo de árvore do chá, óleo essencial de erva-de-limão e óleo de origanum) nas próteses dentárias. Essa revisão demonstrou que a adição de agentes antifúngicos é efetiva, com efeitos mínimos nas propriedades e mecânicas no material.

Enxaguantes bucais incluindo diversos principios ativos (óleos essenciais, clorexidina, cloreto de cetilpiridinio) é extensivamente utilizados na clínica odontólogica ${ }^{53}$. Alguns contêm 5\%-25\% de álcool, que é usado para estabilizar e dissolver os principios ativos com a finalidade de proporcionar refrescância, aumento no prazo de validade e previnir a contaminação por microrganismos. Dados da literatura relataram que há possiveis efeitos colaterais na cavidade oral do indivíduo que utiliza enxaguantes à base de álcool. Além disso, é descrito que o uso desses enxaguantes é proibido para crianças, gestantes lactantes, diabéticos, pacientes com xerostomia e imunocomprometidos. Alguns desses quadros são condições fundamentais para a origem das candidoses ${ }^{53}$. Assim, Maekawa et al. ${ }^{53}$ realizaram um estudo que avaliou a atividade antimicrobiana de novos enxaguantes sem álcool com digluconato de clorexidina frente à $C$. albicans. Nesse estudo, dois enxaguantes bucais sem etanol (Cariax ${ }^{\circledR}$, com $0,12 \%$ de clorexidina e fluoreto de sódio $0,05 \%$; Orthokin ${ }^{\circledR}$, com $0,06 \%$ de clorexidina, fluoreto de sódio $0,05 \%$ e acetato de zinco $0,34 \%$ ) foram comparados com o controle positivo (Periogard ${ }^{\circledR}$, clorexidina $0,12 \%$, com etanol). Cariax ${ }^{\circledR}$ revelou atividade fungicida sobre $C$. albicans semelhante à do controle positivo, mas menor ação fungistática em comparação ao Periogard ${ }^{\circledR}$. Esses autores sugeriram que a utilização de enxaguantes bucais sem álcool ainda é limitada em casos em que há proibição dos produtos convencionais.

Embora agentes antifúngicos comumente usados para tratar o EP, como nistatina (na forma de pastilha e suspensão oral) e fluconazol (comprimido via oral), sejam eficazes no alívio dos sinais e sintomas clínicos da infecção por Candida, a repetição da infecção após o tratamento tem sido comumente reportada. Além disso, a disseminação do uso de agentes antifúngicos resultou no desenvolvimento de espécies resistentes ${ }^{54}$.

Desse modo, na Tabela 1 foi realizada uma pesquisa sistemática na tentativa de avaliar os 
Tabela 1: Tratamentos in vitro/in vivo para a eliminação do biofilme fúngico nas próteses dentárias.

\begin{tabular}{|c|c|c|}
\hline Metodologia aplicada & Resultados & Referência \\
\hline $\begin{array}{l}\text { Avaliação do potencial dos tratamentos plasmáticos } \\
\text { para modificar a química da superfície e a } \\
\text { hidrofobicidade de uma resina de base dentária } \\
\text { ("argon atmosphere" a } 50 \text { W [grupo Ar } 50 \text { W]; } \\
\text { "atmospheric air" a } 130 \text { W [grupo AAt/130 W] na } \\
\text { redução da adesão de C. glabrata. }\end{array}$ & $\begin{array}{l}\text { Tratamentos AAt/130W modificaram a } \\
\text { composição química, hidrofobicidade } \\
\text { e topografia da superfície acrílica. O } \\
\text { grupo Ar/50 W apresentou uma adesão } \\
\text { significativamente menor de C. glabrata } \\
\text { do que o grupo controle, na ausência de } \\
\text { saliva. }\end{array}$ & Zamperini et al. ${ }^{55}$ \\
\hline $\begin{array}{l}\text { O fosfato catiônico e absorvente visível de } \\
\text { absorção de luz (iii) ftalocianinas (GaPcs) } \\
\text { substituídas perifericamente com quatro e } \\
\text { oito grupos metilpiridiloxi foram sintetizados e } \\
\text { investigados como sensibilizadores fotodinâmicos } \\
\text { antimicrobianos. }\end{array}$ & $\begin{array}{l}\text { A GaPc2 substituída com octa- } \\
\text { metilpiridiloxi solúvel em água teve } \\
\text { um valor potencial para o tratamento } \\
\text { fotodinâmico de biofilmes de } C \text {. albicans } \\
\text { formados na resina acrílica dentadura. }\end{array}$ & Mantareva et al. ${ }^{56}$ \\
\hline
\end{tabular}

Foram confeccionadas resinas acrilicas de saliva e inoculados com células de C. albicans durante $1 \mathrm{~h}\left(37^{\circ} \mathrm{C}\right)$. Os testes de Candidacidal foram realizados em biofilmes de $48 \mathrm{~h}$. A adesão celular foi comparada em discos pré-revestidos com $0,12 \%$ de gluconato de clorexidina, 50 microM Hst 5 (histatina) ou 0,6 microM hBD-3 (defensinas) após 24,48 e 72 h de crescimento.

Modificações de superfície com tratamentos plasmáticos com diferentes hidrofobicidades ( $\mathrm{Ar} / 50 \mathrm{~W}, \mathrm{ArO}_{2} / 70 \mathrm{~W}, \mathrm{AAt} / 130 \mathrm{~W}$ ) ou com fluoreto incorporado (Ar/SF $60 \mathrm{~W}$ ) foram avaliados para a redução à aderência de $\mathrm{C}$. albicans.

Os biofilmes expostos nas próteses de 30 pacientes foram coletados e os indivíduos foram separados por grupo: Grupo 1, no qual os pacientes tiveram sua prótese maxilar em microondas durante $3 \mathrm{~min}$ (650W); Grupo 2, no qual pacientes os tiveram sua prótese maxilar em microondas durante 2 min $(650 \mathrm{~W})$. As amostras de biofilme foram coletadas com swab antes e depois da irradiação com microondas.

A Hst 5 (histatina) inibiu significativamente o desenvolvimento do biofilme em $72 \mathrm{~h}$. Por outro lado a clorexidina a $0,12 \%$ inibiu Pusateri et al. ${ }^{57}$ significativamente o desenvolvimento do biofilme em todos os intervalos de tempo.

Os tratamentos com plasma foram eficazes na modificação da superfície do polimetilmetacrilato.
Zamperini et al. ${ }^{7}$

A irradiação de microondas durante 3 min (grupo 1) resultou na esterilização de todas as dentaduras. O grupo 2 apresentou uma diminuição significativa em Candida spp. A irradiação de Ribeiro et al. ${ }^{58}$ microondas por 3 min pode ser um tratamento para prevenir a contaminação cruzada.
Os corpos de provas foram confeccionados com um revestimento de prótese de poli (metacrilato de metila) e os biofilmes de C. albicans ou C. glabrata foram formados na superfície durante $48 \mathrm{~h}$ e os tratamentos de limpeza foram aplicados: dois peróxidos alcalinos (durante 3 ou $15 \mathrm{~min}$ ), 0,5\% de hipoclorito de sódio (10 $\mathrm{min}$ ) ou água destilada (controle, $15 \mathrm{~min}$ ). Logo, as células de biofilme foram quantificadas (célula/mL).

Avaliação da influência da saliva artificial na formação de biofilmes por C. albicans. Grupos estudados: (A) saliva artificial I (carboximetilcelulose); saliva artificial II (glicose oxidase, lactoferrina, lisozima e lactoperoxidase); grupo controle (água destilada estéril).

Nesse estudo in vitro foi utilizada uma coloração diferencial e microscopia eletrônica de varredura (SEM) para avaliar o efeito do hipoclorito de sódio e do gluconato de clorhexidina na viabilidade, remoção e morfologia do biofilme de C. albicans em prótese acrílica.
Os tratamentos da prótese alcalina apresentaram desempenho de limpeza semelhante e ambos diferiram do controle. O hipoclorito de sódio foi o tratamento que eliminou o biofilme e não foram encontradas células vivas após o seu uso.
Vieira et al. ${ }^{59}$

Houve uma diminuição significativa de $29,89 \%$ (1,45 unidades formadoras de colônia [UFC] $/ \mathrm{mL}$ ) de C. albicans na saliva I em relação à saliva $\mathrm{II}(p=0,002$, considerando $p \leq 0,05)$.

As amostras contendo hipoclorito de sódio são a primeira escolha para a limpeza de próteses dentárias em relação a $4 \%$ de clorhexidina. Isso porque essas soluções não apenas destruiram os

Silva et al. ${ }^{60}$ biofilmes de $C$. albicans como também possibilitaram a remoção na resina acrílica termopolimerizada. 


\begin{tabular}{lll}
\hline \multicolumn{1}{c}{ Metodologia aplicada } & \multicolumn{1}{c}{ Resultados } & \multicolumn{1}{c}{ Referência } \\
\hline & Foi evidenciado que os mutantes efg1 & -/- e bcr1 -/- obtiveram uma redução \\
& no crescimento de biofilme com pouco \\
& ou nenhuma matriz extracelular. A \\
& quantificação da carga fúngica mostrou \\
Foi utilizado um modelo estabelecido de EP em & diminuição da colonização ao longo do \\
animais imunocompetentes com a finalidade de & período de infecção em dentaduras e & Yano et al. ${ }^{61}$ \\
determinar o papel da formação de biofilmes no & palatos de ratos inoculados com efg1 -/-, & \\
dano da mucosa durante a patogênese de C. & entretanto não para bcr1 -/- (em relação \\
albicans/ou mutantes defeituosos na morfogênese & aos controles). Em suma, a formação de \\
(efg1 - / -) ou formação de biofilme (bcr1-/-). & biofilmes está aliada ao dano tecidual \\
& durante a EP e que Efg1 e Bcr1 em C. & \\
& albicans são essenciais na patogênese \\
& do EP.
\end{tabular}

Avaliação do efeito da dentadura embebidas pelo ácido benzóico frente a colonização de C. albicans em comparação com gluconato de clorexidina $0,2 \%$ $\left(\right.$ Zordyl $\left.^{\circledR}\right)$. grupo (1): pacientes que utilizaram ácido benzóico imersão das próteses dentárias; grupo (2): pacientes utilizaram zordil (gluconato de clorexidina $(0,2 \%)$ para imersão em prótese

Avaliação do efeito do adesivo, do limpador, da clorexidina e escovação da prótese dentária frente ao biofilme de $\mathrm{C}$. albicans desenvolvido em uma superfície acrílica para evitar a EP.
Esse estudo verificou que a utilização de ácido benzóico em prótese tem um efeito de inibição contra a colonização de $C$. albicans nas superfícies dentaduras mais eficazes que gluconato de clorexidina $0,2 \%$.

O adesivo de dentadura apresenta alta adesividade de $C$. albicans à superfície da dentadura. Entretanto, o limpador e a clorexidina diminuem a adesividade. Em suma, a clorexidina inibe extensivamente a aderência de $C$. albicans sobre a superfície da prótese.

\section{L. rhamnosus e L. casei exibiram} atividade antifúngica contra blastoconidios e biofilme de C. albicans. Nenhum dos
Lactobacillus casei. Os biofilmes de L. rhamnosus e probióticos influenciou na rugosidade
L. casei foram formados e tratados com C. albicans. superficial da resina da base da

Lactobacillus casei. Os biofilmes de L. rhamnosus e probióticos influenciou na rugosida
L. casei foram formados e tratados com C. albicans. superficial da resina da base da A rugosidade da superfície dentária foi avaliada dentadura.

$\operatorname{Arafa}^{62}$

Gantait et al. ${ }^{63}$

Song e Lee ${ }^{64}$ com um microscópio de força atômica.

Avaliação dos efeitos das proantocianidinas (isoladas a partir de frutos de cranberry) de arando de tipo A (AC-PACs) nas propriedades patogênicas e na resposta inflamatória das células epiteliais orais induzidas por $\mathrm{C}$. albicans. Foram utilizadas microplacas para determinar o efeito de AC-PAC sobre o crescimento de C. albicans, bem como a formação de biofilmes corados com cristal violeta. $A$ adesão de $C$. albicans às células epiteliais orais e a discos de resina acrílica foi monitorada por fluorometria.

O polimetil-metacrilato (PMMA) tem sido extensivamente empregado como base de uma resina acrílica de dentadura devido às suas excelentes propriedades físicas e mecânicas. Nesse estudo foi avaliada a adição de grupos fosfato com concentrações de $0 \%, 5 \%, 15 \%$, $10 \%, 20 \%$ e $25 \%$ ao PMMA para melhorar a adsorção de antimicrobianos salivares e inibir a adesão microbiana de C. albicans na superfície do polímero.
AC-PACs não afetaram o crescimento de $C$. albicans, impedindo a formação de biofilmes e diminuindo a adesão de $C$. albicans a células epiteliais orais e discos de resina acrílica revestidos com saliva.
Feldman et al. ${ }^{65}$

A presença de fosfato $\geq 15 \%$ em PMMA reduziu significativamente a adesão mediada por saliva de C. albicans.
Raj e Dentino ${ }^{66}$ 
Tabela 1: Continuação....

\begin{tabular}{|c|c|c|}
\hline Metodologia aplicada & Resultados & Referência \\
\hline $\begin{array}{l}\text { Avaliação da susceptibilidade do biofilme de } \\
\text { C. albicans e C. glabrata, em seus estágios } \\
\text { intermediários e de maturação, à influência das } \\
\text { nanopartículas de prata (silver nanoparticles, SN). } \\
\text { Essas suspensões foram usadas para tratar os } \\
\text { biofilmes de Candida durante } 5 \text { h, foram cultivadas } \\
\text { em superfícies acrílicas no tempo de } 24 \text { h (estágio } \\
\text { intermediário) e } 48 \text { h (estágio de maturação), e sua } \\
\text { eficácia foi determinada pela biomassa total (cristal } \\
\text { violeta) e pela quantificação das UFC. }\end{array}$ & $\begin{array}{l}\text { SNs proporcionaram diminuições } \\
\text { significativas na biomassa total e no } \\
\text { número de UFC de biofilmes de Candida. } \\
\text { Quanto aos estágios intermediários e } \\
\text { de maturação, não houve diferenças } \\
\text { significativas na biomassa total do } \\
\text { biofilme. }\end{array}$ & Monteiro et al. ${ }^{67}$ \\
\hline
\end{tabular}

Avaliação da eficácia de um modelo acrílico de poli (etil metacrilato)/tetrahidrofurfuril metacrilato (PEM/THFM) embebido com fluconazol (FLU) ou clorexidina $(\mathrm{CHX})$ na prevenção da formação de biofilme de Candida in vitro. Discos de PEM/ THFM embebidos com CHX $(n=50)$ ou FLU ( $n$ $=50)$ e discos controle $(n=50)$ foram incubados com C. Albicans por 2, 7, 14, 21 ou 28 dias a 37 ${ }^{\circ} \mathrm{C}$. A biomassa total do biofilme e a atividade metabólica foram quantificadas em cada ponto do tempo utilizando o cristal violeta e XTT ([2,3-bis(2methoxy-4-nitro-5-sulfo-phenyl)-2H-tetrazolium-5carboxanilide]).

Avaliação da inativação fotodinâmica antimicrobiana (antimicrobial photodynamic inactivation, API) mediada por curcumina em biofilmes multiespécies de C. albicans, C. glabrata e Streptococcus mutans de diferentes idades. As amostras de acrílico ( $n=$ 480 ) foram produzidas e incubadas com bactérias e leveduras durante 24 ou 48 h. A API foi realizada com curcumina e luz LED. As amostras de acrílicos adicionais foram tratadas apenas com curcumina ou com luz LED. Amostras de controle positivo não receberam luz e nem curcumina. Após a API, foi realizada a quantificação das $\mathrm{UFC} / \mathrm{mL}$, avaliação do metabolismo celular (XTT), e a biomassa total do biofilme (cristal violeta).

Avaliação do efeito inibitório de partículas de nano-quitosano em resinas acrílicas na formação de biofilme de espécies de Candida. As resinas acrílicas contendo as partículas de nano-quitosano nas concentrações de $0,1 \%, 5 \%$ e $10 \%$ foram colocadas juntas à suspensão de células de Candida isoladas da boca dos indivíduos e a formação de biofilme nas resinas foi medida e comparada.

Avaliação do efeito inibitório da thimoquinona embebida no material de base de prótese de polimetilmetacrilato frente a C. albicans. Oitenta corpos de prova foram divididos em oito grupos com as seguintes concentrações de thimoquinona: $0,5 \%, 1 \%, 1,5 \%, 2 \%, 2,5 \%, 3 \%$ e $5 \%$ de pó acrílico.

Avaliação de uma formulação de enxaguatório bucal contendo 1-n-hexadecil-3-metilimidazólio cloreto $\left(\mathrm{C}_{16} \mathrm{MImCl}\right)$, contra biofilme de Candida spp.

Avaliação da atividade do miconazol em biofilmes maduros de Candida (C. albicans, C. glabrata, C. tropicalis e C. parapsilosis) in vitro formados em discos de PMMA.
Os discos FLU apresentaram inibição reduzida, com inibição metabólica e biomassa média de $12,6 \%$ e $8,8 \%$, respectivamente. Os discos contendo $\mathrm{CHX}$ foram consideravelmente eficazes, inibindo de forma significativa o desenvolvimento do biofilme em $75 \%$ e seu metabolismo em $84 \%$.
Os biofilmes com idade de 24 e $48 \mathrm{~h}$ foram suscetíveis à API mediada por curcumina, embora o biofilme de 24 $\mathrm{h}$ tenha sido mais sensível do que o biofilme de $48 \mathrm{~h}$.
Salim et al. ${ }^{68}$

\section{Quishida et al. ${ }^{69}$}

As partículas de nano-quitosano nas

resinas acrílicas reduziram a formação de Sadeghi et al. ${ }^{70}$ biofilme de espécies de Candida.
A resina acrílica contendo thimoquinona foi eficaz na prevenção da adesão a $C$. albicans.
Al-Thobity et al. ${ }^{71}$

Ação antibiofilme efetiva da formulação

contendo $\mathrm{C}_{16} \mathrm{MImCl}$ frente aos isolados de Bergamo et al. ${ }^{40}$ Candida spp.

Miconazol apresentou alta atividade antifúngica contra os biofilmes de Candida desenvolvidos na superfície de Gebremedhin et al. ${ }^{72}$ 
diversos métodos e tratamentos in vitrolin vivo diferentes dos tratamentos convencionais que vêm sendo estudados para a eliminação do biofilme fúngico nas próteses dentárias.

Em suma, a EP ocasionada nas próteses dentárias possui extensa relação com a adesão dos microrganismos na forma de biofilme. Nesse estudo observamos que $C$. albicans é o principal colonizador de próteses dentárias, mas vem ocorrendo um aumento extensivo de CNA em relação à etiologia desse processo. Os tratamentos convencionais para a EP são capazes de minimizar a sintomatologia típica. Porém, devido aos casos de resistência dos microrganismos associados a essa patologia, a terapêutica pode-se mostrar ineficaz em muitos casos. Nesse sentido, a busca por novas opções de agentes antifúngicos e formulações faz-se de extrema importância.

\section{Conflitos de Interesse}

Os autores declaram não ter conflitos de interesse.

\section{REFERÊNCIAS}

1. Marra J, Perez LEC, Henriques TE, Pinheiro MT, Castro FLA. Avaliação da correlação entre o grau de instruções e qualidade de higiene de usuários de próteses totais com a presença de estomatite protética. Rev Odontol Bras Central. 2017;26:15-20.

2. Melo IA, Guerra RC. Candidíase oral: um enfoque sobre a estomatite por prótese. Rev Salusvita. 2014;33:389414.

3. Dağistan $\mathrm{S}$, Aktas $\mathrm{AE}$, Caglayan F, Ayyildiz A, Bilge M. Differential diagnosis of denture-induced stomatitis, Candida, and their variations in patients using complete denture: a clinical and mycological study. Mycoses. 2009;52(3):266-71. http://dx.doi.org/10.1111/j.14390507.2008.01592.x. PMid:18643887.

4. Arruda CNF, Salles MM, Badaró MM, Oliveira VC, Macedo AP, SilvaLovato $\mathrm{CH}$, et al. Effect of sodium hypochlorite and Ricinus communis solutions on control of denture biofilm: a randomized crossover clinical trial. J Prosthet Dent. 2017;117(6):729-34. http://dx.doi. org/10.1016/j.prosdent.2016.08.035. PMid:27927282.

5. Pereira-Cenci T, Del Bel Cury AA, Crielaard W, Ten Cate JM. Development of candida-associated denture stomatitis: new insights. J App/ Oral Sci. 2008;16(2):8694. http://dx.doi.org/10.1590/ S1678-77572008000200002. PMid:19089197.

6. Yoshijima Y, Murakami K, Kayama S, Liu D, Hirota K, Ichikawa $\mathrm{T}$, et al. Effect of substrate surface hydrophobicity on the adherence of yeast and hyphal Candida.

Mycoses. 2010;53(3):221-6.

http://dx.doi.org/10.1111/j.1439-

0507.2009.01694.x. PMid:19671080.

7. Zamperini CA, Machado AL, Vergani CE, Pavarina AC, Giampaolo ET, da Cruz NC. Adherence in vitro of Candida albicans to plasma treated acrylic resin: effect of plasma parameters, surface roughness and salivary pellicle. Arch Oral Biol. 2010;55(10):763-70. http://dx.doi. org/10.1016/j.archoralbio.2010.06.015. PMid:20667522.

8. Park SE, Blissett R, Susarla SM, Weber HP. Candida albicans adherence to surface-modified denture resin surfaces. $J$ Prosthodont. 2008;17(5):365-9. http://dx.doi.org/10.1111/j.1532849X.2007.00292.x. PMid:18266657.

9. Redding S, Bhatt B, Rawls HR, Siegel G, Scott K, Lopez-Ribot J. Inhibition of Candida albicans biofilm formation on denture material. Oral Surg Oral Med Oral Pathol Oral Radiol Endod. 2009;107(5):669-72. http://dx.doi. org/10.1016/j.tripleo.2009.01.021. PMid:19426921.

10. Agostinho ACMG, Campos ML, Silveira JLGC. Edentulismo, uso de prótese e autopercepção de saúde bucal entre idosos. Rev Odontol UNESP. 2015;44(2):74-9. http://dx.doi. org/10.1590/1807-2577.1072.

11. Ribeiro AFL, Leal MCC, Marques APO. Importance of geriatric dentistry to elderly nutrition. Rev Gaucha Odontol. 2012;60:241-6.

12. Kumaresan GD, Subha M. Burning mouth syndrome. Int J Orofac Biol. 2017;1:53-8.
13. Matthews DC, Clovis JB, Brillant MGS, Filiaggi MJ, McNally ME, Kotzer RD, et al. Oral health status of long-term care residents: a vulnerable population. J Can Dent Assoc. 2012;78:c3. PMid:22364866.

14. Mujica V, Rivera H, Carrero M. Prevalence of oral soft tissue lesions in an elderly venezuelan population. Med Oral Patol Oral Cir Bucal. 2008;13(5):E270-4. PMid:18449108.

15. Bianchi CM, Bianchi HA, Tadano T, Paula CR, Hoffmann-Santos HD, Leite $\mathrm{DP} J \mathrm{R}$, et al. Factors related to oral candidiasis in elderly users and nonusers of removable dental prostheses. Rev Inst. Med Trop. 2016;58:17. http://dx.doi.org/10.1590/S16789946201658017.

16. Avrella D, Goulart LS. Isolamento de Candida spp. da mucosa oral de pacientes submetidos ao tratamento quimioterápico. Rev. Bras. Anál. Clín. 2008;40:205-7.

17. Sesma N, Morimoto S. Estomatite protetica: etiologia, tratamento e aspectos clínicos. JBB. 2011;2:24-9.

18. Corte-Real I, Figueiral M, Reis Campos J. As doenças orais no idoso: considerações gerais. Rev Port Estomatol Med Cir Maxilofac. 2011;52:175-80.

19. Mima EG, Pavarina AC, Ribeiro DG, Dovigo LN, Vergani CE, Bagnato VS. Effectiveness of photodynamic therapy for the inactivation of Candida spp. on dentures: in vitro study. Photomed Laser Surg. 2011;29(12):82733. http://dx.doi.org/10.1089/ pho.2011.3022. PMid:21916614. 
20. Giannini PJ, Shetty KV. Diagnosis and management of oral candidiasis. Otolaryngol Clin North Am 2011;44(1):231-40. http://dx.doi. org/10.1016/j.otc.2010.09.010.

21. Newton A. Denture sore mouth: a possible etiology. $\mathrm{Br}$ Dent $\mathrm{J}$. 1962;112:357-60.

22. Arnaud RR, Soares MSM, Santos MGC, Santos RC. Estomatite protética: prevalência e correlação com idade e gênero. Rev. Bras. Ciênc. Saúde. 2012;16(1):59-62. http://dx.doi. org/10.4034/RBCS.2012.16.01.09.

23. Scalercio M, Valente T, Israel MS, Ramos ME. Estomatite protética versus candidíase: diagnóstico e tratamento. RGO. 2007;55:395-8.

24. Maddi A, Scannapieco F. Oral biofilms, oral and periodontal infections, and systemic disease. Am J Dent. 2013;26(5):249-54. PMid:24479275.

25. Prakash B, Shekar M, Maiti B, Karunasagar I, Padiyath S. Prevalence of Candida spp. among healthy denture and nondenture wearers with respect to hygiene and age. J Indian Prosthodont Soc. 2015;15(1):2932. http://dx.doi.org/10.4103/09724052.155041. PMid:26929483.

26. Koch C, Bürgers R, Hahnel S. Candida albicans adherence and proliferation on the surface of denture base materials. Gerodontology. 2013;30(4):309-13. http://dx.doi.org/10.1111/ger.12056. PMid:24117985.

27. Cuéllar-Cruz M, López-Romero E, Villagómez-Castro JC, Ruiz-Baca E. Candida species: new insights into biofilm formation. Future Microbiol. 2012;7(6):755-71. http:// dx.doi.org/10.2217/fmb.12.48. PMid:22702528.

28. Silva MP, Chibebe J JR, Jorjão AL, Machado AK, Oliveira LD, Junqueira $\mathrm{JC}$, et al. Influence of artificial saliva in biofilm formation of Candida albicans in vitro. Braz Oral Res. 2012;26(1):248. http://dx.doi.org/10.1590/S180683242012000100005. PMid:22344334

29. Thein ZM, Samaranayake YH, Samaranayake LP. In vitro biofilm formation of Candida albicans and non-albicans Candida species under dynamic and anaerobic conditions. Arch Oral Biol. 2007;52(8):7617. http://dx.doi.org/10.1016/j. archoralbio.2007.01.009. PMid:17321487.

30. Rodger G, Taylor RL, Pearson GJ, Verran J. In vitro colonization of an experimental silicone by Candida albicans. J Biomed Mater Res B Appl Biomater. 2010;92(1):226-35. http:// dx.doi.org/10.1002/jbm.b.31509. PMid:19921746.

31. Coulthwaite L, Verran J. Potential pathogenic aspects of denture plaque. Br J Biomed Sci. 2007;64(4):180-9. http://dx.doi.org/10.1080/09674845.200 7.11732784. PMid:18236742.

32. Czerninski R, Pikovsky A, Gati I, Friedman M, Steinberg D. Comparison of the efficacy of a novel sustained release clotrimazole varnish and clotrimazole troches for the treatment of oral candidiasis. Clin Oral Investig. 2015;19(2):467-73. http://dx.doi. org/10.1007/s00784-014-1259-5. PMid:24867230.

33. Nevzatoğlu EU, Ozcan M, Kulak-Ozkan Y, Kadir T. Adherence of Candida albicans to denture base acrylics and silicone-based resilient liner materials with different surface finishes. Clin Oral Investig. 2007;11(3):231-6. http:// dx.doi.org/10.1007/s00784-007-01063. PMid:17333305.

34. Al-Rifaiy MQ. The effect of mechanical and chemical polishing techniques on the surface roughness of denture base acrylic resins. Saudi Dent J. 2010;22(1):13-7. http://dx.doi. org/10.1016/j.sdentj.2009.12.006. PMid:23960474

35. Subramani K, Jung RE, Molenberg A, Hammerle $\mathrm{CH}$. Biofilm on dental implants: a review of the literature. Int J Oral Maxillofac Implants. 2009;24(4):616-26. PMid:19885401.

36. Ataídes FS, Abrão FY, Costa CR, Silva MRR, Pimenta FC, Palos MAP, et al. Identificação de espécies de Candida em saliva de profissionais da saúde. Rev. Eletr. Enf. 2010;12(3):498501. http://dx.doi.org/10.5216/ree. v12i3.6472.

37. Shinawi LA. The effect of various denture cleansers on the colour stability of different denture base resins. IJPRAS. 2017;6:238-46.

38. Borghi E, Morace G, Borgo F, Rajendran R, Sherry L, Nile C, et al. New strategic insights into managing fungal biofilms. Front Microbiol. 2015;6:1077. http://dx.doi.org/10.3389/ fmicb.2015.01077. PMid:26500623.

39. Ramage G, Rajendran R, Sherry L, Williams C. Fungal biofilm resistance. Int J Microbiol. 2012;2012:528521. http://dx.doi.org/10.1155/2012/528521. PMid:22518145.
40. Bergamo VZ, Donato RK, Nemitz MC, Acasigua GA, Selukar BS, Lopes W, et al. Assessing an imidazolium salt's performance as antifungal agent on a mouthwash formulation. $J$ Appl Microbiol. 2016;121(6):1558-67. http://dx.doi.org/10.1111/jam.13283. PMid:27569114.

41. Harrison JJ, Ceri H, Yerly J, Rabiei M, $\mathrm{Hu} Y$, Martinuzzi R, et al. Metal ions may suppress or enhance cellular differentiation in Candida albicans and Candida tropicalis biofilms. Appl Environ Microbiol. 2007;73(15):4940-9. http://dx.doi.org/10.1128/AEM.0271106. PMid: 17557844.

42. Mathé L, Van Dijck P. Recent insights into Candida albicans biofilm resistance mechanisms. Curr Genet. 2013;59(4):251-64. http://dx.doi. org/10.1007/s00294-013-0400-3. PMid:23974350.

43. Silva S, Negri M, Henriques M, Oliveira R, Williams DW, Azeredo J. Adherence and biofilm formation of non-Candida albicans Candida species. Trends Microbiol. 2011;19(5):241-7. http:// dx.doi.org/10.1016/j.tim.2011.02.003. PMid:21411325.

44. Albuquerque P, Casadevall A. Quorum sensing in fungi: a review. Med Mycol. 2012;50(4):337-45. http://dx.doi.or g/10.3109/13693786.2011.652201. PMid:22268493.

45. Ten Cate JM, Klis FM, Pereira-Cenci T, Crielaard W, de Groot PW. Molecular and cellular mechanisms that lead to Candida biofilm formation. J Dent Res. 2009;88(2):105-15. http://dx.doi. org/10.1177/0022034508329273. PMid:19278980.

46. Pathak AK, Sharma S, Shrivastva P. Multi-species biofilm of Candida albicans and non-Candida albicans Candida species on acrylic substrate. J Appl Oral Sci. 2012;20(1):70-5. http://dx.doi.org/10.1590/S167877572012000100013 . PMid:22437681.

47. Valentini F, Luz MS, Boscato N, Pereira-Cenci T. Biofilm formation on denture liners in a randomised controlled in situ trial. $J$ Dent. 2013;41(5):420-7. http://dx.doi. org/10.1016/j.jdent.2013.02.012. PMid:23454226.

48. Sánchez-Vargas LO, Estrada-Barraza D, Pozos-Guillen AJ, Rivas-Caceres R. Biofilm formation by oral clinical isolates of Candida species. Arch Oral Biol. 2013;58(10):1318-26. http://dx.doi. org/10.1016/j.archoralbio.2013.06.006. PMid:23849353. 
49. Oliveira RC, Brum SC, Oliveira RS, Goyatá FR. Aspectos clínicos relacionados à estomatite protética. Int J Dent. 2007;6(2):51-4.

50. Skupien JA, Valentini F, Boscato N, Pereira-Cenci T. Prevention and treatment of Candida colonization on denture liners: a systematic review. J Prosthet Dent. 2013;110(5):356-62. http://dx.doi. org/10.1016/j.prosdent.2013.07.003. PMid:23998622.

51. Peixoto JV, Rocha MG, Nascimento RTL, Moreira VV, Kashiwabara TGB. Candidíase-uma revisão de literatura. BJSCR. 2014;8:75-82.

52. Iqbal Z, Zafar MS. Role of antifungal medicaments added to tissue conditioners: a systematic review. J Prosthodont Res. 2016;60(4):2319. http://dx.doi.org/10.1016/j. jpor.2016.03.006. PMid:27085676.

53. Maekawa LE, Brighenti FL, Lamping $\mathrm{R}$, de Oliveira LD, Marcacci S, Kogaito CY. Atividade antimicrobiana de enxaguatórios bucais sem álcool a base de clorexidina sobre Candida albicans. Rev Odontol UNESP. 2010;39:15-9.

54. Pfaller MA. Antifungal drug resistance: mechanisms, epidemiology, and consequences for treatment. $\mathrm{Am}$ J Med. 2012;125(1 Suppl):S3-13. http://dx.doi.org/10.1016/j. amjmed.2011.11.001. PMid:22196207.

55. Zamperini CA, Carneiro HL, Rangel EC, Cruz NC, Vergani CE, Machado AL. In vitro adhesion of Candida glabrata to denture base acrylic resin modified by glow-discharge plasma treatment. Mycoses. 2013;56(2):13444. http://dx.doi.org/10.1111/j.14390507.2012.02223.x. PMid:22809146.

56. Mantareva V, Kussovski V, Angelov I, Wöhrle D, Dimitrov R, Popova $\mathrm{E}$, et al. Non-aggregated $\mathrm{Ga}$ (III)phthalocyanines in the photodynamic inactivation of planktonic and biofilm cultures of pathogenic microorganisms. Photochem Photobiol Sci. 2011;10(1):91-102. http:// dx.doi.org/10.1039/B9PP00154A. PMid:21031201.

57. Pusateri CR, Monaco EA, Edgerton M. Sensitivity of Candida albicans biofilm cells grown on denture acrylic to antifungal proteins and chlorhexidine. Arch Oral Biol.
2009;54(6):588-94. http://dx.doi. org/10.1016/j.archoralbio.2009.01.016. PMid:19249746.

58. Ribeiro DG, Pavarina AC, Dovigo LN, Palomari Spolidorio DM, Giampaolo ET, Vergani CE. Denture disinfection by microwave irradiation: a randomized clinical study. J Dent. 2009;37(9):66672. http://dx.doi.org/10.1016/j. jdent.2009.04.009. PMid:19481327.

59. Vieira AP, Senna PM, Silva WJ, Del Bel Cury AA. Long-term efficacy of denture cleansers in preventing Candida spp. biofilm recolonization on liner surface. Braz Oral Res. 2010;24(3):342-8. http://dx.doi.org/10.1590/S180683242010000300014 . PMid:20877973.

60. Silva PMB, Acosta EJTR, Pinto LR, Graeff M, Spolidorio DMP, Almeida RS, et al. Microscopical analysis of Candida albicans biofilms on heat-polymerised acrylic resin after chlorhexidine gluconate and sodium hypochlorite treatments. Mycoses. 2011;54(6):e712-7. http://dx.doi. org/10.1111/j.1439-0507.2010.02005.x. PMid:21605193.

61. Yano J, Yu A, Fidel PL JR, Noverr MC. Transcription factors Efg1 and Bcr1 regulate biofilm formation and virulence during candida albicans-associated denture stomatitis. PLoS One. 2016;11(7):e0159692. http://dx.doi. org/10.1371/journal.pone.0159692.

62. Arafa KAO. Efficacy of benzoic acid denture cleansing against the colonization of Candida albicans. Saudi Journal for Health Sciences. 2016;5(2):76-81. http://dx.doi. org/10.4103/2278-0521.193002.

63. Gantait S, Bhattacharyya J, Das S, Biswas S, Ghati A, Ghosh S, et al. Comparative assessment of the effectiveness of different cleaning methods on the growth of Candida albicans over acrylic surface. Contemp Clin Dent. 2016;7(3):336-42. http:// dx.doi.org/10.4103/0976-237X.188554. PMid:27630498.

64. Song YG, Lee SH. Inhibitory effects of Lactobacillus rhamnosus and Lactobacillus casei on Candida biofilm of denture surface. Arch Oral Biol. 2017;76:1-6. http://dx.doi. org/10.1016/j.archoralbio.2016.12.014. PMid:28063305.

65. Feldman M, Tanabe S, Howell A, Grenier D. Cranberry proanthocyanidins inhibit the adherence properties of Candida albicans and cytokine secretion by oral epithelial cells. BMC Complement Altern Med. 2012;12:6.

66. Raj PA, Dentino AR. New phosphated poly(methyl methacrylate) polymers for the prevention of denture-induced microbial infection: an in vitro study. Clin Cosmet Investig Dent. 2011;3:2532.

67. Monteiro DR, Takamiya AS, Feresin LP, Gorup LF, Camargo ER, Delbem AC, et al. Susceptibility of Candida albicans and Candida glabrata biofilms to silver nanoparticles in intermediate and mature development phases. J Prosthodont Res. 2015;59(1):428. http://dx.doi.org/10.1016/j. jpor.2014.07.004. PMid:25168655.

68. Salim N, Silikas N, Satterthwaite JD, Moore C, Ramage G, Rautemaa R. Chlorhexidine-impregnated PEM/ THFM polymer exhibits superior activity to fluconazole-impregnated polymer against Candida albicans biofilm formation. Int $J$ Antimicrob Agents. 2013;41(2):193-6. http://dx.doi. org/10.1016/j.ijantimicag.2012.09.006. PMid:23127479.

69. Quishida CC, Mima EGO, Jorge JH, Vergani CE, Bagnato VS, Pavarina AC. Photodynamic inactivation of a multispecies biofilm using curcumin and LED light. Lasers Med Sci. 2016;31(5):997-1009. http://dx.doi. org/10.1007/s10103-016-1942-7. PMid:27126412.

70. Sadeghi Ardestani Z, Falahati M, Sayah Alborzi S, Ashrafi Khozani M, Rostam Khani F, Bahador A. The effect of nanochitosans particles on Candida biofilm formation. Curr Med Mycol. 2016;2(2):28-33. http://dx.doi. org/10.18869/acadpub.cmm.2.2.1. PMid:28681017.

71. Al-Thobity AM, Al-Khalifa KS, Gad MM, Al-Hariri M, Ali AA, Alnassar T. In vitro evaluation of the inhibitory activity of thymoquinone in combatting Candida albicans in denture stomatitis prevention. Int J Environ Res Public Health. 2017;14(7):743. http://dx.doi. org/10.3390/ijerph14070743.

72. Gebremedhin S, Dorocka-Bobkowska B, Prylinski M, Konopka K, Duzgunes N. Miconazole activity against Candida biofilms developed on acrylic discs. J Physiol Pharmacol. 2014;65(4):593600. PMid:25179092. 